\title{
Removing Redundancy Point Clouds Based on Multi-view Geometric
}

\author{
Junmin LV \\ Center for Information Technology \\ Tian Fu College of SWUFE \\ Mianyang China \\ 49263957@qq.com \\ Jin Guo * \\ Center for Information Technology \\ Tian Fu College of SWUFE \\ Mianyang China \\ 860018662@qq.com \\ * Corresponding Author
}

\author{
Xiaoning Chen \\ Center for Information Technology \\ Tian Fu College of SWUFE \\ Mianyang China \\ 408169569@qq.com \\ Hongyan Xu \\ Center for Information Technology \\ Tian Fu College of SWUFE \\ Mianyang China \\ xia438@163.com
}

\begin{abstract}
According to the problem of structure light measuring for registration, such as the low efficiency of three-dimensional modeling because of overlapping points, a removing redundancy points technology based on multiepipolar line resolution had been explored out. The traditional method belonged to the post-processing point cloud. This algorithm was based on uniqueness of object point in multiview geometric constraint before the point cloud come out. First, the multiple view of the camera was unified to the global coordinate system. Then the threedimensional point clouds after calculating was been on the back projection. The distance between the two points that was greater than the given threshold would be reserved and the projection residual larger point would be reserved to avoid redundant point generation. Experiment proves that the algorithm proposed can removing redundancy points effectively and improving the efficiency of three-dimensional modeling without reducing the scanning density and accuracy, detailed information of the surface with large curvity can also be preserved.
\end{abstract}

Keywords-structure light scanning; redundancy points; registration; multiview geometric; $S V D$

\section{INTRODUCTION}

A 3-dimensional (3D) structured light measurement system based on multi-frequency phase shift needs a number of stitching measurements in order to get the global point cloud(Global point cloud is the point cloud with a unified coordinate system) of big objects that spatially span a wide range. When multiple point clouds measured in different times are stitched into a complete point cloud, there are often large quantities of redundant point clouds in the overlapping area between two original point clouds. The data amount of redundant point clouds becomes larger and larger with the increasing of stitching times, which affects the ensuing processing of $3 \mathrm{D}$ point cloud data and reduces the speed and accuracy of $3 \mathrm{D}$ modeling.
Deleting the redundant points in the overlap area is always after the three-dimensional point cloud generation. Liang, et al. adopted a normal constrained point cloud fusion method which largely depends on the choice of seed points [2]. Cao, et al. suggested using moving least square for multi-view cloud data fusion. However, their algorithm is still time-consuming [3]. Du proposed a mixed mode to refine point clouds [4]. Yang adopted a multi-resolution simplification algorithm, which is also computationally intensive [5]. Nira Dyn, et al. constructed a non-negative function which is to be used as a metric number according to the importance of points. Then the point clouds are simplified by using iterative methods. Likewise, the method is considered inefficient in speed. Alexa, et al. suggested calculating the effect of sample points on the moving surfaces of least square to simplify point clouds. However, the method is complex because it involves nonlinear optimization [7]. The above-mentioned methods are based on 3D point clouds and use data structures such as octree. Remove redundant point clouds by calculating the information such as curvatures and densities is computationally intensive and inefficient. Moreover, information such as the original scanned data is not guaranteed.

This study avoided the usage of traditional methods in the post-processing of point clouds. The uniqueness principle of the measurement object points and the correspondence between $3 \mathrm{D}$ point clouds and the 2dimensional (2D) images in multi-views are utilized to automatically calculate the overlapping areas of the stitching and the previously measured areas. The $2 \mathrm{D}$ points in overlapping areas are not used for matching reconstruction, which avoids the generation of redundant data points in overlapping areas from the source.

\section{REDUNDANT POINT DELETING METHOD}

A 3D structured light measurement system based on binocular vision uses "Zhang Zhengyou" flat calibration to 
calibrate the cameras which is crossed and obtains the internal parameters (central cooradinate of the image, focal distance, distortion coefficient) and external parameters (translation matric, rotation matrix) of the cameras. A world coordinate system is established for the left camera which coincides with the camera's own coordinate system [9]. The internal parameters of the left camera and the right camera are coincident, while the coordinate systems of the external parameters are not. However, the transformation matrix between two measurements can be calculated according to the labels on objects, or by the unlabeled stitching method. Then the external camera parameters which are scanned in different times can be unified into the same coordinate system. The parameters are then optimized by bundle adjustment. The redundant points are removed according to multi-epipolar constraints.

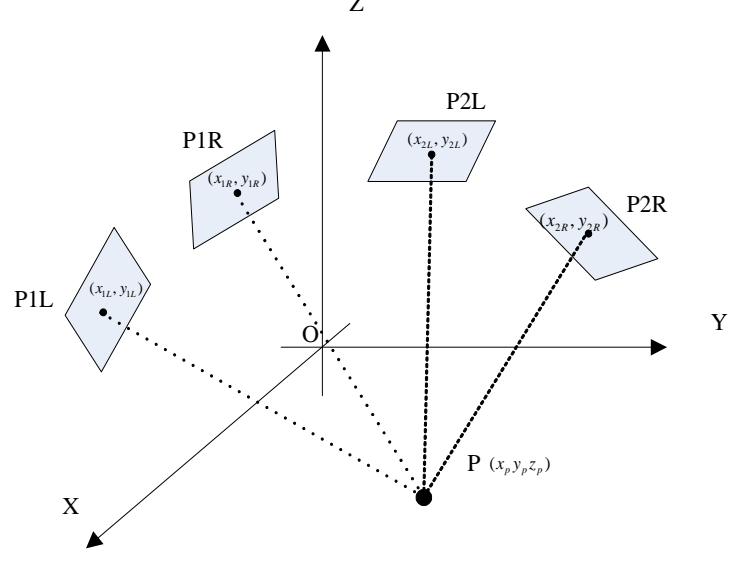

Figure 1. The geometric constraints of multi-view geometric

As shown in Fig .1, there is a unified world coordinate system OXYZ. P1L and P1R are the image points of $\mathrm{P}$ in the left camera and the right camera in the first measurement, respectively. $\mathrm{P} 2 \mathrm{~L}$ and $\mathrm{P} 2 \mathrm{R}$ are the image points of $\mathrm{P}$ in the left camera and the right camera in the second measurement, respectively. Using the geometric relationship of a spatial triangle and the calibrated camera parameters, the $3 \mathrm{D}$ coordinates of $\mathrm{P},\left(x_{p} y_{p} z_{p}\right)$, can be calculated from the image points in the first measurement, $P 1 L\left(x_{1 L}, y_{1 L}\right)$ and $P 1 L\left(x_{1 R}, y_{1 R}\right)$. Similarly, the 3D coordinates of $\mathrm{P},\left(x_{p}^{\prime} y_{p}^{\prime} z_{p}^{\prime}\right)$, can be calculated from the image points in the second measurement, $P 2 L\left(x_{2 L}, y_{2 L}\right)$ and $P 2 R\left(x_{2 R}, y_{2 R}\right)$. The two measurements are for the same spatial point $\mathrm{P}$; therefore, a redundant point is generated by calculating the two $3 \mathrm{D}$ coordinates $\left(x_{p} y_{p} z_{p}\right)$ and $\left(x_{p}^{\prime} y_{p}^{\prime} z_{p}^{\prime}\right)$. The image of cameras is reversible. Project the point $\left(x_{p}^{\prime} y_{p}^{\prime} z_{p}^{\prime}\right)$ is back to the left camera in the first measurement. If the back-projection point is on the imaging surface and the point is already used in the first measurement, the point $\left(x_{p}^{\prime} y_{p}^{\prime} z_{p}^{\prime}\right)$ is redundant and can be deleted. For areas with large curvature changes, the errors of back-projection are calculated to decide whether to delete or not.

\section{A. Unifying external parameters of multi-view cameras}

The transformation matrix $M$ is solved by SVD decomposition. The coordinate system of the first measurement is $O_{1} X_{1} Y_{1} Z_{1}$, and projection matrices of the left and the right camera are $P M_{1 L}$ and $P M_{1 R}$. The coordinate system of the second measurement is $\mathrm{O}_{2} \mathrm{X}_{2} \mathrm{Y}_{2} \mathrm{Z}_{2}$, and projection matrices of the left and the right camera are $P M_{2 L}$ and $P M_{2 R}$, and the transformation matrix $\mathrm{M}$ between two measurements can be solved by SVD analysis [10]:

$$
\left[\begin{array}{llll}
x_{0} & y_{0} & z_{0} & 1
\end{array}\right]^{T}=M\left[\begin{array}{llll}
x_{1} & y_{1} & z_{1} & 1
\end{array}\right]^{T}
$$

Transform the projection matrix of the second measurement into $O_{1} X_{1} Y_{1} Z_{1}$ :

$$
\begin{aligned}
& P M_{2 L}^{\prime}=P M_{2 L} * M^{-1} \\
& P M_{2 R}^{\prime}=P M_{2 R} * M^{-1}
\end{aligned}
$$

The coordinates of the point clouds calculated from $P M_{2 L}^{\prime}$ and $P M_{2 R}^{\prime}$ are unified into $O_{1} X_{1} Y_{1} Z_{1}$

\section{B. Calculating 3D points in overlapping areas}

In structured light measurement based on binocular cameras, the higher frequency of the spatial measurement object is sampled with, the denser the obtained $3 \mathrm{D}$ points are and the better the object details can be reflected. However, in the stitching measurement process, the sampling frequency of the same space in two measurements can be different due to the camera shooting angles. Two or more spatial points in an image may be back-projected to one point, even though they don't overlap in space. Therefore, when 3D points in the image overlapping areas are calculated, images with high spatial sampling frequency is needed to be reserved to guarantee the preservation of details. Moreover, when the spatial sampling frequencies are closed, the accuracies of the 3D points of the overlapping areas calculated in different views are different. In order to increase the accuracy of point clouds after deleting redundant points, the errors of back-projection are required to compare with reserve points with high accuracy in two scans. The process method for points with high curvatures and high accuracy is as follows.

Define the unit plane of an object surface as a square with its side length being $\varepsilon$. $\varepsilon$ is the average 3D point distance in the central area of the image plane when the surface is scanned. In this study, $\mathcal{E}$ is set to $0.02 \mathrm{~mm}$. Define the unit plane of the image plane as a square with its side length being $e=1$, representing a unit pixel. Let the pixel in the left image in the first measurement, $P_{11}$, correspond to $3 \mathrm{D}$ point $P_{1}^{\prime}$, and the back-projection error of it is e1. Make the pixel in the left image be in the second measurement, $P_{21}$, correspond to $3 \mathrm{D}$ point $P_{1}^{\prime \prime}$, and the back-projection error of it be e2. Project $P_{1}^{\prime \prime}$ back to the right image of the first scan, obtaining the back-projection image point $P_{11}^{\prime}$. If $\left\|P_{11}-P_{11}^{\prime}\right\| \leq e$ and $\left\|P_{1}^{\prime}-P_{1}^{\|}\right\| \geq \varepsilon$, the pixel distance between the obtained points, $P_{1}^{\prime}$ and $\stackrel{P}{1}^{\prime \prime}$, is not longer than $e$. However, the two points belong to different unit planes of the object, therefore they should be reserved. If $\left\|P_{11}-P_{11}^{\prime}\right\| \leq e$ and $\left\|P_{1}^{\prime}-P_{1}^{\prime \prime}\right\|<\varepsilon, P_{1}^{\prime}$ and $P_{1}^{\prime \prime}$ are two points on the same plane which are close to each other. Therefore, only one of them should be reserved. In order to reserve the point with higher accuracy, e 1 and $\mathrm{e} 2$ are compared. If e1<e2, $P_{1}^{\prime}$ is reserved; otherwise, $P_{1}^{\prime \prime}$ is reserved. 


\section{Algorithmic process of removing redundant points in overlapping areas caused by multiple scans}

When global stitching scan is applied to relatively big objects, with the increasing of scan numbers, the redundant point clouds in common areas will also increase, the removal algorithm for the redundant point in overlapping areas is as follows:

Step 1: The first measurement need not redundant point removal. Calculate the projection matrices $P M_{1 L}$ and $P M_{1 R}$, and obtain 3D point clouds. Label the point involved in the first calculation as $\operatorname{mask}[i][u][v]$, indicating whether the point with coordinates $(u, v)$ in the $i$-th scan is involved in 3D point calculation. For the first measurement, set i to 1.

Step 2: From the second stitching measurement on, match the 3D labeled point, compute the transformation matrix of this measurement, $M_{2}$.

Step 3: Unify the coordinate systems of the projection matrices of the left and the right cameras in the second measurement using the transformation matrix, obtaining $P M_{2 L}^{\prime}$ and $P M_{2 R}^{\prime}$.

Step 4: Calculate the 3D point cloud data $Q_{2}(x, y, z)$ from the projection matrices $P M_{2 L}^{\prime}$ and $P M_{2 R}^{\prime}$. Then project the 3D point cloud data back to the left camera in the first measurement using the method introduced in Section 1.2. Label the points involved in the second calculation as $\operatorname{mask}[i]\left[u^{\prime}\right]\left[v^{\prime}\right]$.

Step 5: Repeat Step 2 to Step 4 for the i-th stitching measurement. Project the data of the i-th measurement back to the left camera in the first, second, ..., and the (i1)-th measurements.

\section{This algorithm has the following advantages}

- The overlapping areas in stitching measurement can be automatically recognized. Only the redundant points in overlapping areas are processed, and the measuring accuracy and the sampling density are not damaged. In overlapping parts, unit spaces are defined. Only one 3D point in a unit space is reserved. That is, only one calculation operation is performed for this point. When calculating a 3D point, neither the camera parameters nor the original image matching relationship is changed. Therefore the calculated 3D point and the point of the original point cloud are correspondent, which makes the quality of the original point cloud undamaged.

- The overall accuracy of point clouds is improved. In structured light scanning systems, the accuracy of point clouds is affected by camera calibration accuracy and image matching accuracy, etc. Binocular back-projection error can quantitatively measure the error of the triangle formed by the image point of the dual camera image plane, the optical center and the object point. That is to say, theoretically, the combined effects of all kinds of errors on reconstruction can be measured. In this study, this index is used to remove the low-accuracy points in overlapping areas and reserve high-accuracy points. Therefore the overall quality of point clouds is theoretically improved, maximizing the effect of redundant point removal. Divide the point clouds of two scans on the image, and choose and reserve those with high accuracy in the unit plane.
- There are two core points in the algorithm. The first is to identify the overlapping areas. The second is to perform a single object point calculation. The calculation of the two core points needs the projection matrix of the dual cameras in each scan in global coordinate system. Moreover, whatever stitching method needs, the calculation of the projection matrix of the current image is in the global coordinate system. Therefore the proposed algorithm is suitable for prevalent stitching methods. Due to the characters of structured light measurement, the proposed method can also be applied to measurements of big objects, such as vehicles. Combined with photogrammetric systems and registering the single point clouds with global labeling points, the overlapping point clouds are avoided and the data redundancy is largely reduced, which improve the efficiency of 3D modeling.

\section{EXPERIMENTAL VERIFICATION}

The 3D light measuring system established in this study consists of a raster projector (Mitsubishi DX-320 models of projectors with a resolution of $1024 * 768$ and a brightness of 2500 lumens), two Daheng industrial cameras with Schneider lens (resolution of $1280 * 1024$, focal length of $12 \mathrm{~mm}$ ), a calibration plate, a computer and a tripod.

Firstly, the 3D measuring system is calibrated to obtain the internal and external parameters of the cameras. The pasted circle centers are used as marker points. Two stitching measurements are performed on a jar, according to the multi-frequency heterodyne principle. Negative exponential sequence expansion is adopted for solving the phase of the point clouds [11][12]. The $\mathrm{M}$ matrix for matching the marker points of circle centers is calculated by using SVD method. The marker points in overlapping areas are recognized and removed by using the proposed algorithm. Fig. 2(a) shows the enlarged overlapping parts of the point clouds in two measurements. It can be seen from the figure that the dark part around the mark circle with a large number of redundant point clouds is the overlapping part after stitching. Fig.2(b) shows the removal effect of overlapping points using the proposed method. It can be seen from Fig.2(b) that the effect is maximized. The above experiment verifies the effectiveness of the proposed method for removing redundant points of overlapping areas. Comparing the images in Fig.2, it can be concluded that the point cloud can still describe the 3D morphology of the object.

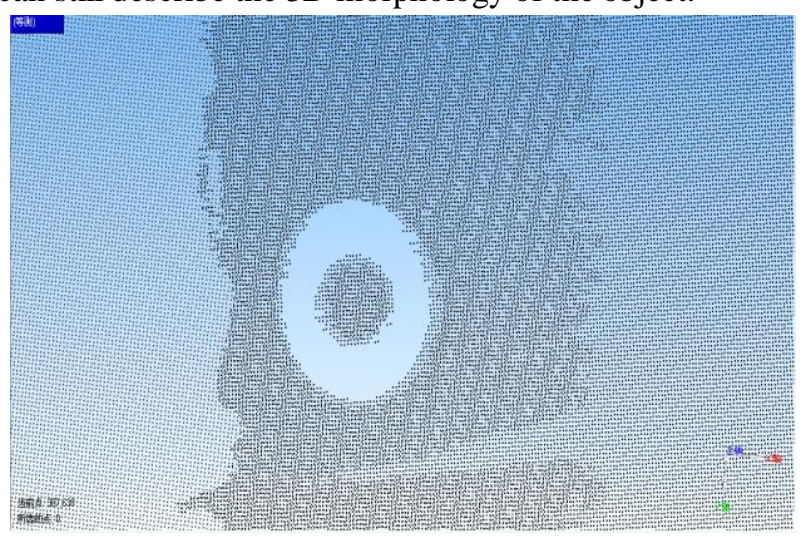

(a) Overlapping point clouds after stitching 


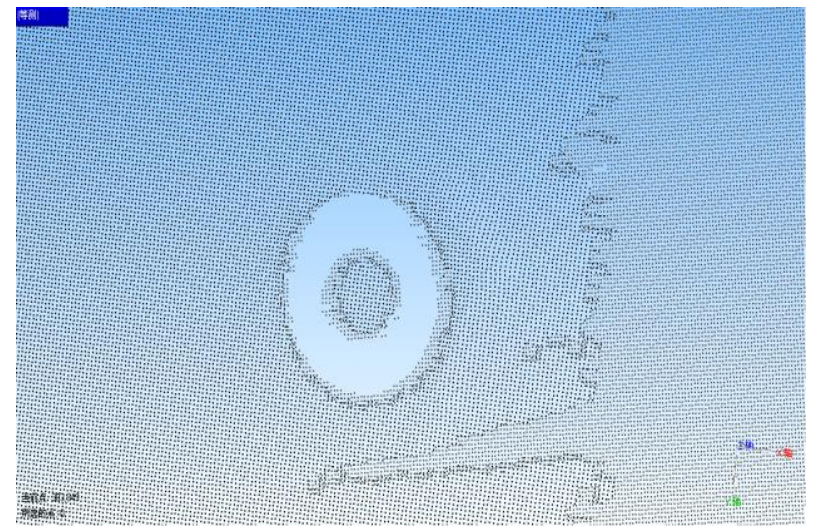

(b)Point cloud after overlapping part is deleted

Figure 2. overlapping points cloud delete effect comparison icon

To further verify the performance of the proposed method, a part of the point cloud in the overlapping area is selected. Then compare the traditional removing algorithm for redundant point cloud with the proposed method. Results produced by the proposed method are compared with the outcome taken from uniform sampling and curvature sampling using Geomagic Studio. The region of the measured jar with maximum curvature change is triangulated for comparison. It can be seen from Table 1 that the proposed method achieves the best removing effect [13]. Fig.4(a) shows the original point cloud with a large number of redundant points. A large number of redundant triangles also exist after triangularization, which is shown in Fig.5(a). Fig.4(b) and 4(c) show the point cloud after unified sampling and curvature sampling, respectively. Although redundant points can be reduced, they cannot be eliminated. Fig.5(b) and 5(c) are the results of triangularization. After using the two methods, the point clouds are not smooth. Fig.4(d) shows the result of the proposed method. It can also be known from Table 1 that the redundant point removing efficiency of the proposed

method is relatively high. Fig.5 (d) shows the result of triangularization after the proposed method is used. The smoothness, point density and detail description ability are very close. Fig. 3 shows the triangularization result of the measured object after using the proposed method to delete redundant point clouds. The point cloud shown in Fig.3 is smooth. In summary, the proposed method can balance the redundant point removal efficiency and the quality of obtained clouds. The method does not only have high efficiency of redundancy deletion, but also maintains the original detail information of objects.

TABLE I. COMPARATIVE RESULTS OF PROPOSED METHOD AND OTHER ALGORITHMS

\begin{tabular}{|l|l|l|l|}
\hline & \multicolumn{1}{|c|}{$\begin{array}{c}\text { No. of } \\
\text { points }\end{array}$} & $\begin{array}{c}\text { No. of } \\
\text { triangular facets }\end{array}$ & deletion efficiency \\
\hline $\begin{array}{l}\text { original } \\
\text { point cloud }\end{array}$ & 771587 & 1505485 & $0.0 \%$ \\
\hline $\begin{array}{c}\text { Unified } \\
\text { sampling }\end{array}$ & 673399 & 1320076 & $12.7 \%$ \\
\hline $\begin{array}{c}\text { Curvature } \\
\text { sampling }\end{array}$ & 643762 & 1199481 & $16.5 \%$ \\
\hline proposed & 561081 & 1099498 & $27.3 \%$ \\
\hline
\end{tabular}

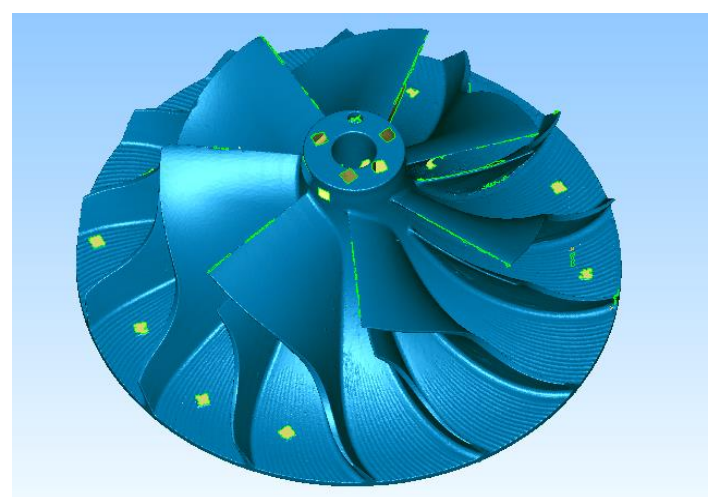

Figure 3. Triangularization after overlapping point cloud deletion
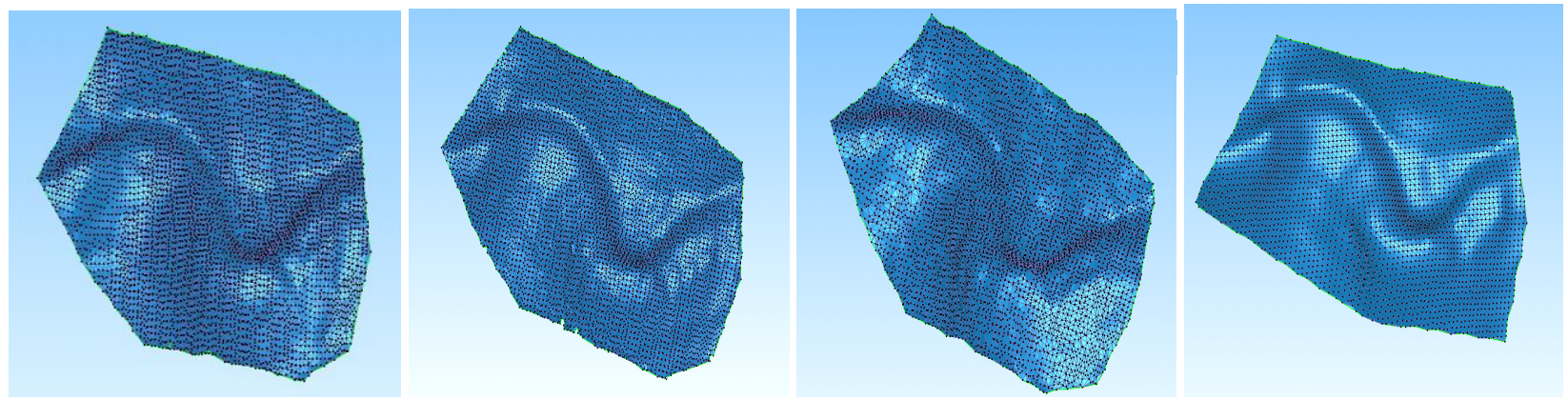

(a) original point clouds (b) uniform sample (c) curvature sample (d) the algorithms sample

Figure 4. Point cloud Comparison of different algorithms in high curvature places 

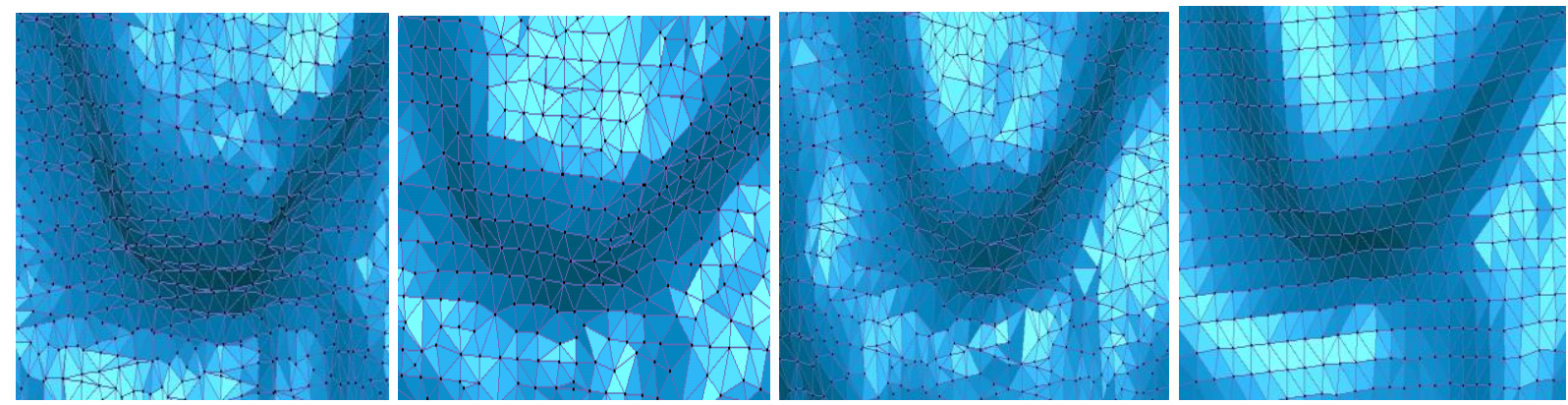

(a) triangulate the original point clouds (b) triangulate the uniform sample(c) trangulate the curvature sample (d) trangulate the algorithms sample

Figure 5. Triangularization comparison of different algorithms in high curvature places

\section{CONCLUSIONS}

Traditional point cloud processing algorithms are generally used after point clouds are generated, including point cloud denoising and smoothing, overlapping point cloud deletion, and triangularization, etc. Establishing the topology structures of point clouds is required and is computationally intensive. The proposed overlapping point cloud deleting method uses the multi-view constraints and the uniqueness principle of object points before the structured measuring system obtains $3 \mathrm{D}$ point clouds. The proposed method deletes overlapping point clouds from the source and avoids the generation of redundant points. Experiments show that the method can effectively remove redundant points and better preserve object details in multiple stitching measurements, despite planes or curved surfaces, provided that the accuracy of a single measurement and the point cloud density is guaranteed.

\section{REFERENCES}

[1] YANG Ying-bao,LIU Xian-yong,YANG Jun-ping. Point-clouds registration algorithm based on improve projection[J].Application Research of Computers,2011, 28(10):3954-3956

[2] LIANG Xin-he,LIANG Jin,GUO Cheng, et al.Multi Range Images Integration Algorithm Based on Normal Constraint[J].Journal of Xi an Jiaotong University,2009, 43(5):71-75

[3] CAO Ju-ming, WU SHOUR-Slam, LIANG Jin,et al. Moving Least Squares Based Incremental Multi-View Range Images Integration Algorithm[J].Journal of Xi an Jiaotong University,2009,43(9): 4650
[4] DU Xiao-hui,YIN Bao-cai,KONG De-hui.Mixed simplification algorithm of point clouds[J]. Computer Engineer ing and Applications, 2007,43(34):43-45

[5] YANG Bin,FAN Yuan-yuan,WANG Ji-dong. Multi-resolution simplification algorithm for point cloud[J].Journal of Computer Applications,2011, 31(10):2717-2720

[6] Dyn N,Iske A,Wendland H.Meshfree thinning of 3D point clouds[J].Foundations of Computational Mathematics, 2008,8(4):409-425

[7] HUANG Wen-ming,PENG Xi-wei,WEN Pei-zhi,et al.Simplification of scattered point cloud with geometric feature reservation[J].Computer Engineering and Applications, 2009,45(28):168-170

[8] WANG Hong-tao,ZHANG Li-yan,DU Ji,LI Zhong -wen,ZHOU Ru-rong.Simplification and Error Analysis Based on Implicit Surface for Measuring Point-sets[J].Journal of Image and Graphics, 2007,12 (11):2114-2118

[9] SHAO Nuan,LI Hui-guang,LIU Le.Binocular stereo distancemeasurement method based on feature matching algorithm[J].Journal of Yanshan University,2012,36(1):57-61

[10] Guo Jin,Liu Xianyong,Chen Xiaoning,Yao Yiyong.An unmarked $3 \mathrm{D}$ point cloud automatic registration technology[J].Computer Applications and Software,2012,29(4):144-147

[11] Xu Wei,Chen Xiaobo,Xi Juntong.A Method of Phase Error Compensation for Structural Light Measurement[J].Acta Optica Sinica,2011,31(3):1-6

[12] LI Lu-Lu,SU Xian-Yu,DOU Yun-Fu,CHEN Feng.Error analysis and algorithm design of temporal phase unwrapping[J].Journal of Sichuan University(Natural Science Edition),2012, 49(1): 102-108

[13] LIN Xiu-fang,Study on data sampling algorithm of reverse engineering[J].Electronic Design Engineering, 2012,6(11):23-26 